\title{
Nouvelles données sur la présence de Colletes marginatus SMITH (Hymenoptera, Colletidae) sur le littoral belge
}

\author{
Par Jelle Devalez * et Nicolas J Vereecken **
}

\section{Introduction}

Colletes marginatus SMITH (Hymenoptera, Colletidae) est une espèce de petite taille $(6-9 \mathrm{~mm})$ qui est présente dans une grande partie de l'Europe occidentale, de la Finlande et la Norvège au nord, à l'Espagne au sud et jusqu'en Mongolie à l'est (Warncke 1978; Else en prép.). Cette espèce est signalée des pays voisins de la Belgique comme l'Allemagne (Westrich 1989), l'Angleterre (Else en prép.), les Pays-Bas (Peeters et al. 1999) ou encore la France (Rasmont et al. 1995), et ce principalement sur les sites côtiers.

En Belgique, l'espèce n'a été recensée qu'à 7 reprises entre 1949 et 1978, notamment dans la région d'Anvers (Antwerpen, nord du pays) et sur le littoral, à Nieuport (Nieuwpoort), (Figure 3). Au cours du mois de juin 2007, l'un d'entre nous (NV) a eu l'occasion d'avoir une correspondance électronique au sujet de la présence probable de cette espèce à la côte belge avec Jeroen de Rond (voir http://www.naturalmedia.nl), un collègue hollandais de la section Hyménoptères de la NEV (Nederlandse Entomologische Vereniging). Ce dernier a eu la gentillesse de nous répondre et nous a fourni de plus amples informations sur les habitats potentiels où l'espèce pourrait être trouvée.

\section{Observations au Zwin}

Au cours du mois de juillet 2007, l'un d'entre nous (JD) s'est rendu dans la réserve naturelle du Zwin à Knokke-Heist pour y observer l'entomofaune caractéristique des milieux côtiers, en particulier les abeilles sauvages. Outre les habitats caractéristiques du cordon dunaire de la côte belge, la réserve naturelle du Zwin comprend de nombreux sites favorables à l'observation de l'avifaune, ce qui en fait l'un des hauts lieux de l'ornithologie en Belgique.

Le 17 juillet 2007, lors d'une prospection sur une dépression dunaire humide à végétation claisemée comprenant notamment des euphraises comme Euphrasia stricta D. WOLFF ex JF LEHM et Odontites vernus (BELLARDI) (Scrophulariaceae) et, sur les parties plus drainées, Senecio jacobea L. et Hieracium sp. (Asteraceae),

\footnotetext{
* Labo voor Zoöfysiologie, Krijgslaan 281 S33 B-9000 Gent,

Belgique. E-mail: jelle.devalez@gmail.com

** Eco-Ethologie Evolutive, Université Libre de Bruxelles CP 160/12, Av. F.D. Roosevelt 50, B-1050 Bruxelles, Belgique.

E-mail: nicolas.vereecken@ulb.ac.be
}

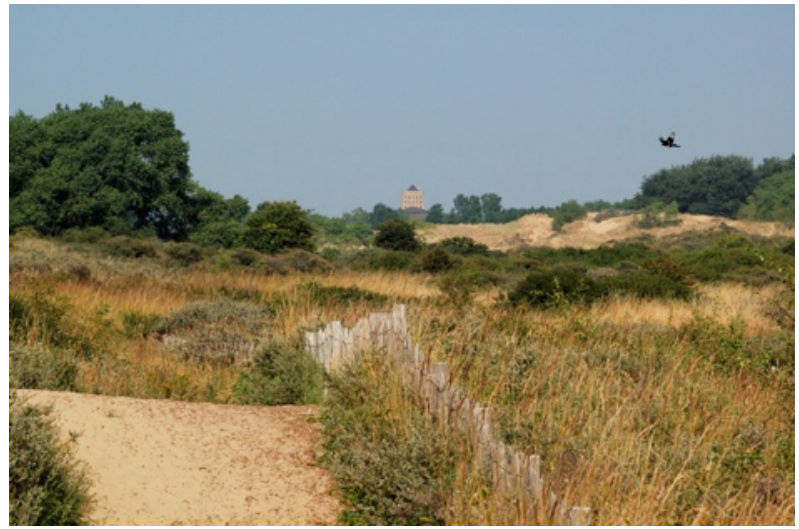

Figure 1. Milieu dunaire de la côte belge (Photo NJ Vereecken)

une femelle identifiée plus tard comme Colletes marginatus SMITH par George Else (Natural History Museum, Londres, Angleterre) et Stuart Roberts (University of Reading, Angleterre) fut observée visitant activement les inflorescences d'E. stricta et d'O. vernus. Ces observations ont été répétées les 18 et 19 juillet sur les mêmes plantes, et des spécimens ont été prélevés pour identification. Il nous semble intéressant de mentionner ici que les femelles n'ont pas été observées en train de récolter du pollen sur ces plantes: il se pourrait donc que les espèces végétales susmentionnées soient exclusivement visitées au cours de vols d'approvisionnement en nectar. Cette hypothèse est confortée par le fait que ni Westrich (1989), ni Else (en prép.) ne mentionnent ces euphraises comme source de pollen. En effet, les femelles de $C$. marginatus sont réputées comme oligolectiques sur diverses Fabacées comme des Medicago (M. sativa), des Melilotus (M. alba, $M$. officinalis) ou encore des Trifolium (T. arvense, T. repens).

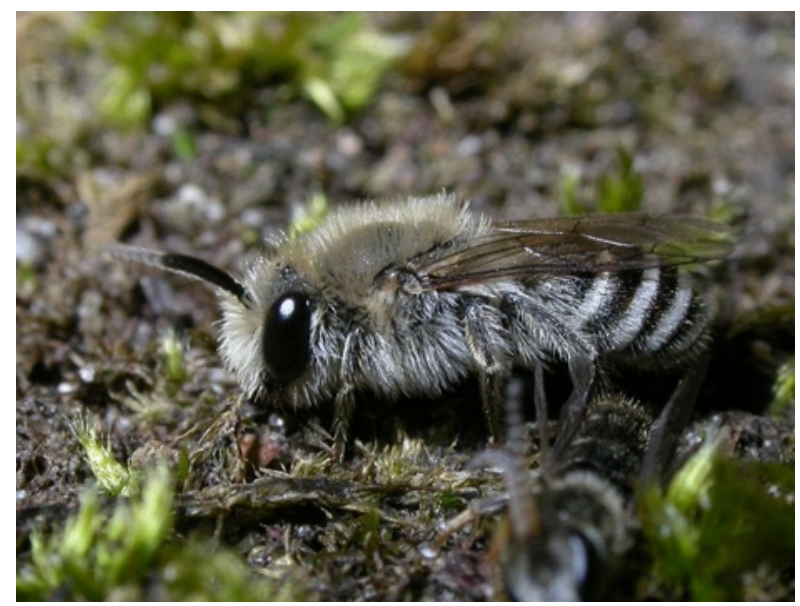

Figure 2. Male de Colletes marginatus SMITH (Photo J. Devalez) 


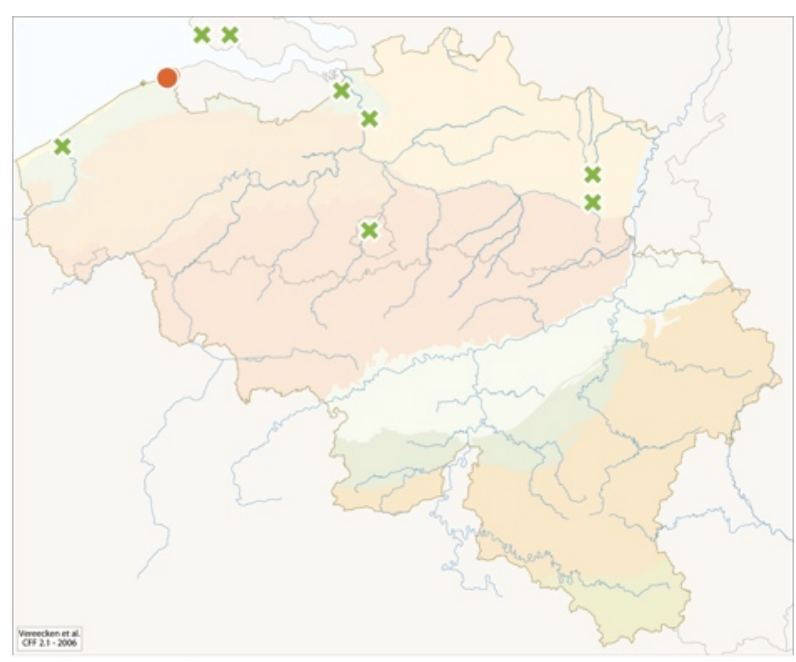

Figure 3. Distribution des observations de Colletes marginatus SMITH en Belgique. Ces données sont issues de la Banque de Données Fauniques Gembloux-Mons (BDFGM) et le site décrit dans cette étude est indiqué en rouge sur la carte

D'autres plantes sont occasionnellement visitées pour le nectar par les mâles et les femelles de C. marginatus: Else (en prép.) signale notamment diverses Apiacées (p.ex. Eryngium maritimum L. Oenanthe lachenalii CG GMEL, Daucus carota L.), Asteracées (Cirsium arvense (L.), Senecio jacobea L.) ou encore des Rosacées (Rubus fruticosus GN JONES). Il est intéressant de noter qu'une des femelles de $C$. marginatus observée au cours de cette étude a été capturée sur $S$. jacobaea, à savoir la même plante que celle sur laquelle les spécimens de la région d'Anvers (Antwerpen) ont été capturés (Figure 3).

Une femelle de l'abeille coucou Epeolus variegatus (L.) (Hymenoptera, Apidae), qui poursuivait régulièrement les femelles de $C$. marginatus a également été observée et collectée au cours de ces prospections. Bien que Westrich (1989) ne mentionne qu'E. cruciger comme abeille coucou pour cette espèce, Richards (1937) et Else (en prép.) citent également $E$. variegatus comme cleptoparasite des nids de C. marginatus.

\section{Conclusion et perspectives}

Ces nouvelles données confirment la présence de $C$. marginatus à la côte belge et apportent des informations complémentaires concernant l'écologie de cette espèce. A titre d'information, nous notons que le dernier spécimen attribué à cette espèce a été capturé il y a pratiquement une trentaine d'années en Belgique. Ces observations offrent de nombreuses perspectives de recherches, en particulier le long de la côte belge, qui présente un réseau de réserves naturelles s'étirant de la frontière française (réserve du Westhoek, à La Panne/ De Panne) jusqu'à la limite des Pays-Bas (réserve du Zwin). La présence de $C$. marginatus dans ces réserves et les milieux environnants devrait être examinée de plus prêt, et divers aspects de l'écologie de cette espèce, comme les choix floraux, la biologie reproductive ou les relations hôtes-parasites, mériteraient une attention toute particulière afin de mieux cerner les facteurs biotiques et abiotiques qui déterminent le maintien de cette espèce dans les milieux naturels du littoral.

\section{Remerciements}

Nous remercions chaleureusement George Else (Natural History Museum, Londres, Angleterre) et Stuart Roberts (University of Reading, Angleterre) qui ont eu la gentillesse de se pencher sur les spécimens collectés pour identification.

\section{Références bibliographiques}

Else GR, (en prépation). The Bees of the British Isles.

Peeters TMJ, Raemakers IP \& Smit J, 1999. Voorlopige atlas van de Nederlandse bijen (Apidae). European Invertebrate Survey - Nederland, Leiden.

Rasmont P, Ebmer PA, Banaszak J \& van der Zanden G, 1995. Hymenoptera Apoidea Gallica. Liste taxonomique des abeilles de France, de Belgique, de Suisse et du Grand-Duché de Luxembourg. Bull. Soc. Entomol. France 100 (HS): 1-98.

Richards OW, 1937. A study of the British species of Epeolus Latr. and their races, with a key to the species of Colletes (Hymen., Apidae). Trans. Soc. Brit. Ent. 4: 89-130.

Warncke K, 1978. Über die westpalärktischen Arten der Bienengattung Colletes Latr. (Hymenoptera, Apoidea). Polski Pismo Ent. 48: 329-370

Westrich P, 1989. Die Wildbienen Baden-Wûrttembergs. Spezieller Teil: Die Gattungen und Arten. Eugen Ulmer GmbH \& Co, Stuttgart.

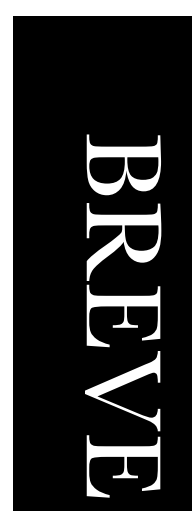

